Rapid Reviews COVID-19

\title{
Review 1: "Detection of SARS-CoV-2 Viral Particles Using Direct, Reagent-Free Electrochemical Sensing"
}

Charles Henry 1

${ }^{1}$ Professor, Colorado State University, Department of Chemistry

Published on: Jan 18, 2021

DOI: 10.1162/2e3983f5.6d92f85f

License: Creative Commons Attribution 4.0 International License (CC-BY 4.0). 


\section{$\underline{\text { RR:C19 Evidence Scale rating by reviewer: }}$}

- Strong. The main study claims are very well-justified by the data and analytic methods used. There is little room for doubt that the study produced has very similar results and conclusions as compared with the hypothetical ideal study. The study's main claims should be considered conclusive and actionable without reservation.

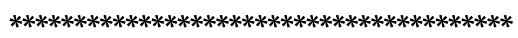

\section{Review:}

This manuscript describes a reagentless electrochemical sensor for detecting intact SARS-CoV-2 virus particles in saliva samples. The sensing concept is based on the bending kinetics of a modified DNA strand attached to an antibody and is highly innovative. The sensor has been previously tested on proteins and is shown here for the detection of an intact viral particle for the first time. Overall, this is a potentially useful and impactful report but the authors should consider the following important points.

1) In the introduction, the authors state that existing reagent free electrochemical systems are not compatible with the detection of intact virus particles. However, there are a number of reports showing just this concept. For example, consider Channon et al, 2018, Analytical Chemistry and Mayall et al, 2020, ACS Sensors.

2) In going from Figure 2 to Figure 3, the time increased from 5 minutes to 10 minutes. Why?

3) The authors report their units in Figure 3 as copies per $\mathrm{mL}$. Standard units are PFU/mL or $\mathrm{TCID} 50 / \mathrm{mL}$. Please correlated the levels to those used by the rest of the field.

4) The authors do their development with recombinant protein and pseudo virus samples. They mention testing with heat treated viruses in S3. I think this is very important data that should be part of the main manuscript since there is a significant difference in recombinant proteins/pseudo virus and inactivated real virus.

5) The authors highlight the negatives of NP swabs and the positives of saliva samples. I think they need to be more careful here. Non-NP swabs are a viable non-invasive sampling method and saliva has it's own problems in terms of complexity, collection (particularly among young and old populations) and difficulties associated with interference from foods, beverages, and pharmaceuticals. This is just a word of caution that should be added that the case is not as clear as made. 
6) For real samples and Figure 4, more information is needed. First, saliva is highly viscous (and of highly variable viscosity). Some discussion of the impact of sample viscosity is needed. Second, the results are plotted as a function of deltaI. More information on the level of SARS in the samples is needed. What were the ct values? 